\title{
Testing gravity at short distances: Gravity Resonance Spectroscopy with qBOUNCE
}

\author{
Tobias Jenke ${ }^{1, a}$, Joachim Bosina ${ }^{1,2}$, Gunther Cronenberg ${ }^{1,2}$, Hanno Filter $^{2}$, Peter Geltenbort ${ }^{1}$, Andrei N. Ivanov ${ }^{2}$, Jakob \\ Micko $^{1,2}$, Mario Pitschmann ${ }^{2}$, Tobias Rechberger ${ }^{2}$, René I.P. Sedmik ${ }^{1}$, Martin Thalhammer ${ }^{1,2}$, and Hartmut Abele ${ }^{2, \mathrm{~b}}$ \\ ${ }^{1}$ Institut Laue-Langevin, 71 avenue des Martyrs, 38000 Grenoble, France \\ ${ }^{2}$ Atominstitut TU Wien, Stadionallee 2, 1020 Wien, Austria
}

\begin{abstract}
Neutrons are the ideal probes to test gravity at short distances - electrically neutral and only hardly polarizable. Furthermore, very slow, so-called ultracold neutrons form bound quantum states in the gravity potential of the Earth. This allows combining gravity experiments at short distances with powerful resonance spectroscopy techniques, as well as tests of the interplay between gravity and quantum mechanics. In the last decade, the $q$ BOUNCE collaboration has been performing several measurement campaigns at the ultracold and very cold neutron facility PF2 at the Institut Laue-Langevin. A new spectroscopy technique, Gravity Resonance Spectroscopy, was developed. The results were applied to test various Dark Energy and Dark Matter scenarios in the lab, like Axions, Chameleons and Symmetrons. This article reviews Gravity Resonance Spectroscopy, explains its key technology and summarizes the results obtained during the past decade.
\end{abstract}

\section{Introduction}

To the best of today's knowledge, all physical processes are based on four fundamental forces: The electromagnetic interaction, the weak and the strong interaction, and gravity. The first three forces are described in terms of quantum field theories, and their most general description is the Standard Model of Particle Physics. Although suspected to be incomplete, most predictions of the Standard Model could be verified, and it has been defying any of the numerous experimental tests and challenges. In particular, the discovery of the Higgs boson $[1,2]$ was a further enormous success. Gravity, on the other hand, the fourth fundamental interaction, is a classical field theory. The idea to describe gravity in the frame of General Relativity (GR) as a consequence of the properties of space-time, is extremely successful: Not a single experimental test could so far find any deviation from GR, and the experimental discovery of Gravitational Waves in 2016 marks a milestone 100 years after the formulation of GR by A. Einstein.

One problem of modern physics is the fact that the Standard Model of Particle Physics and General Relativity have not yet been successfully united. An obvious problem concerns the Hierarchy Problem, which addresses the fact that gravity is so much weaker than the other forces. In order to solve this problem, [3] suggested the existence of large extra dimensions that would couple only to gravity, while the Standard Model effectively deals with our three ordinary spatial dimensions. It turned out that gravity is only weakly tested at short distances, and the authors could

\footnotetext{
a e-mail: jenke@ill.fr

b e-mail: abele@ati.ac.at
}

propose the existence of extra dimensions with a size of mm-range! This proposal lead to a renaissance for gravity experiments at short distances, and it turned out that substantial contributions are coming from measurements with neutrons. Another main motivation for gravity tests with neutrons at short distances comes from Cosmology: the application of GR to the whole Universe, including an astonishingly small number of assumptions, leads to the Standard Model of Cosmology, $\Lambda$ CDM. Numerous observation campaigns pinned down the parameters of the model to the percent level, and revealed a number of cosmological mysteries: many different observations like the measurements of rotation curves of spiral galaxies, gravitational lensing, and the observed anisotropy of the cosmic microwave background point to the existence of an unknown form of invisible, so-called Dark Matter (DM). The observed accelerated expansion of the Universe points to the existence of an additional type of energy, so-called Dark Energy (DE).

Numerous scientific collaborations worldwide try to shed light on these mysteries. Surprisingly, lab experiments on Earth can contribute to these open questions of cosmology or a unification of all four interactions. To do so, they test gravity at short distances, or specific hypothetical models to explain Dark Matter or Dark Energy under well-defined lab conditions. It turns out that neutrons are ideal test particles for such experiments. The reason is that neutrons are electrically neutral and only hardly polarizable.

A very sensitive probe to test gravity at short distances as well as many types of DM and DE scenarios consists of very slow, so-called ultracold neutrons (UCN) placed on horizontal flat surfaces. These neutrons will form quantum mechanical bound states in the gravitational potential of 

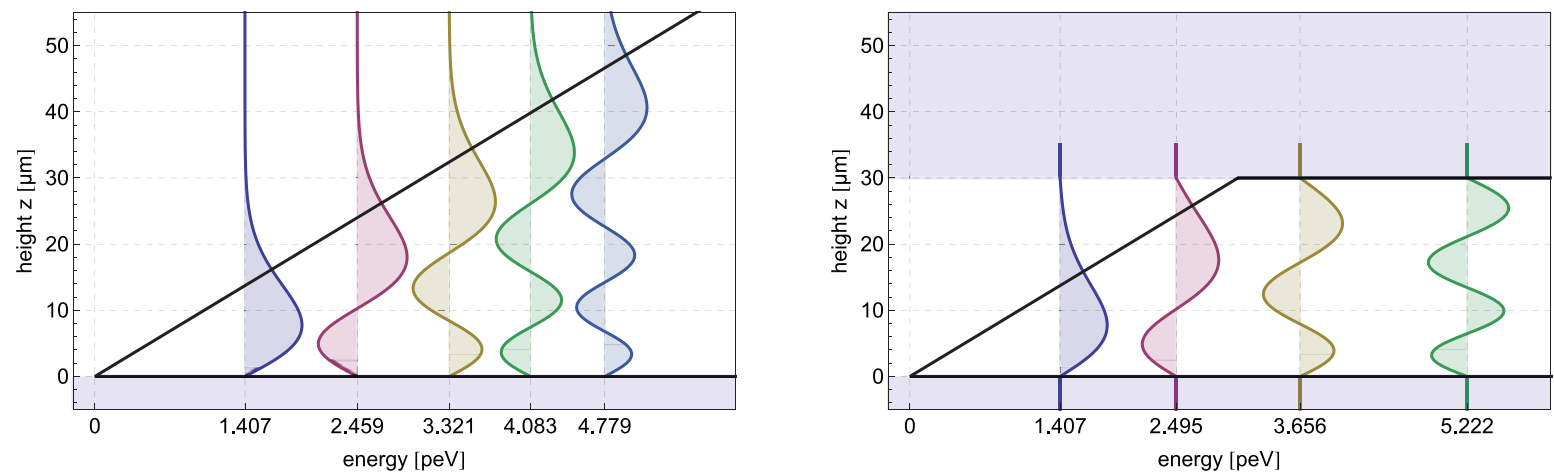

Figure 1. Gravitationally Bounced Quantum States of UCNs. Left side: the plot shows the wave functions of the first five bound quantum states of UCNs in the gravity potential of the Earth above a horizontal flat mirror. The black line depicts the linear gravity potential $m_{g} g z$. Right side: In some cases, it is convenient to place a second boundary condition on top at a well-defined distance. This influences the states and its eigenenergies.

the Earth. The problem of a quantum particle bound in a general linear potential was already discussed in 1928 [4]. In the 1970s, the Schrödinger equation for massive particles in the gravity field was discussed as a textbook example for students [5,6]. Only shortly after the discovery of ultracold neutrons 50 years ago $[7,8]$, gravitational level experiments were suggested [9]. It took roughly 25 years, until an international collaboration reported on the discovery of gravitational bound quantum states of ultracold neutrons [10]. This pioneering work paved the way for numerous future precision experiments.

The Schrödinger equation of a particle on a horizontal flat surface exposed to the linearized gravity potential is equivalent to Airy's differential equation, and the wave functions are given as superposition of Airy functions $A i(z)$. It is convenient to scale the length-, energy- and time axes $z, E$ and $t$ to dimensionless quantities $\tilde{z}, \tilde{E}$ and $\tilde{t}$ in order to result in a dimensionless Schrödinger equation by using the following substitutions:

$$
\begin{aligned}
& z=\tilde{z} \cdot z_{0}, \quad z_{0}=\sqrt[3]{\frac{\hbar^{2}}{2 m_{g} m_{i} g}}, \\
& E=\tilde{E} \cdot E_{0}, \quad E_{0}=m_{g} g z_{0}, \\
& t=\tilde{t} \cdot t_{0}, \quad t_{0}=\frac{\hbar}{E_{0}}
\end{aligned}
$$

Here, the energy scale $E_{0}$, the length scale $z_{0}$ and the time scale $t_{0}$ depend on different combinations of the inertial and gravitational mass of the particle $m_{i}$ and $m_{g}$, the reduced Planck constant $\hbar$, and the local acceleration of Earth $g$.

The first five eigenstates are depicted in Fig. 1. For neutrons, $z_{0}$ has a value of approx. $5.87 \mu \mathrm{m}$, hence the spatial extent of the corresponding probability densities is of the order of several tens of microns. This is wellabove the optical resolution of the human eye (!). The value of $z_{0}$ and $t_{0} \approx 1 \mathrm{~ms}$ allows in principle to measure the time evolution of these states, provided that they can be prepared in some way, that mechanical steps of a few tens of micrometers between flat surfaces can be controlled, and that flat surfaces of a few $\mathrm{cm}$ length can be produced. The system is described in the literature as Quantum Bouncing Ball (QBB) [11]. Precision measurements of its time evolution allow to deduce information about gravity on the level of micrometers by measuring quantum phases. QBB experiments with ultracold neutrons have been performed in the past, but are not subject of this article.

The eigenenergy levels $E_{k}$ of the states are given by the energy scale $E_{0}$ multiplied by the $k$-th zero of the Airy function $\operatorname{Ai}(z)$. They are not equidistant, and the difference between any two eigenenergies is a unique number. Therefore, any two states can be treated as an effective two-level system, provided that the neutron's time of flight in the system $\tau$ is long enough so that the corresponding resonance width $1 / \tau$ does not lead to an overlap with a neighboring resonance. This conclusion has been the basis for the development of Gravity Resonance Spectroscopy (GRS), a resonance method which does not rely on the electromagnetic interaction.

Furthermore, the gravitational and inertial mass enter differently in $z_{0}$ and $E_{0}$. This feature of Schrödinger's equation allows for a test of the weak equivalence principle in the quantum regime: The determination of $z_{0}$ and $E_{0}$ from QBB and GRS experiments and an independent measurement of $g$ lead to an effective test of $m_{i}$ and $m_{g}$ and hence test the weak equivalence principle. This test is conceptually different from classical tests, as it does not rely on a classical trajectory path.

In some situations, it is convenient to place a second horizontal boundary condition on top of the first at a distance of a few tens of microns. This second boundary condition admits as normalizable solution of Schrödinger's equation a linear combination of Airy functions $\operatorname{Ai}(z)$ and $B i(z)$. It also shifts the eigenenergies of the states. The first five eigenstates with a second boundary condition at $l=30 \mu \mathrm{m}$ are depicted in Fig. 1 .

In the following, we will focus on the development of GRS within the $q$ BOUNCE project. Here, we will revisit the key technologies in order to realize our measurements before shortly reviewing their results.

\section{Key technologies to achieve Gravity Resonance Spectroscopy}

The implementation of a resonance spectroscopy technique for gravitationally bound quantum states does not necessarily involve electromagnetic fields and interactions. Therefore, the experimental techniques are rather different 


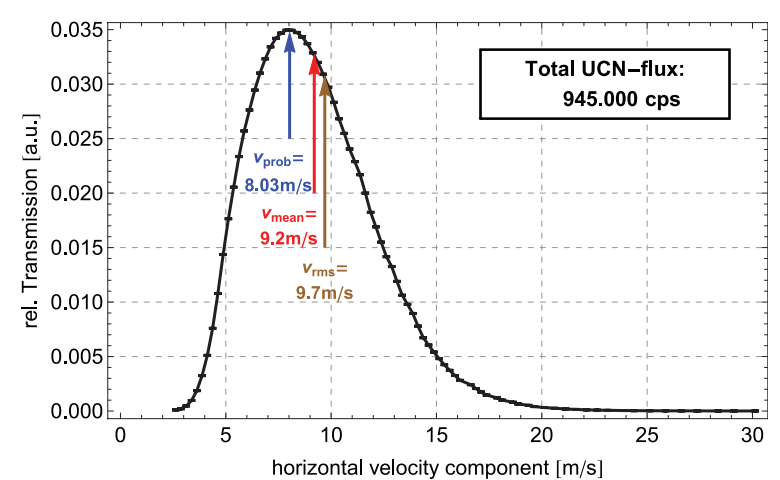

Figure 2. Velocity spectrum of the PF2/UCN beam position. The data was taken before the commissioning of $q$ BOUNCEs' Ramsey spectrometer in 2016. For a full analysis of the data, see [12,13].

from standard experiments with atoms, photons or molecules.

\subsection{Strong UCN source}

As ultracold neutrons do not exist in a lab at a university, the experiment has to take place at an ultracold neutron source. In our case, we use the instrument PF2 at the Institute Laue-Langevin (ILL). Although the UCN flux at PF2 is the highest worldwide, count rates of only a few neutrons per minute are to be expected. Therefore, all experiments are optimized to the peak velocity of the corresponding PF2 beam line, which is roughly $8 \mathrm{~m} / \mathrm{s}$. The corresponding velocity spectrum of the beamline PF2/UCN is depicted in Fig. 2.

\subsection{Horizontal, flat surfaces as boundary conditions}

An obvious need of all experiments are "horizontal, flat surfaces". In our experiments, we use polished BK7 glass substrates with a roughness of $R_{a}<2 \mathrm{~nm}(\mathrm{rms})$ and a waviness of less than $20 \mathrm{~nm}$ over the whole surface. The experiments make use of UCNs on these substrates at grazing incidence. Their Boron content helps to efficiently remove unwanted UCNs (at higher angle of incidence) by absorption. In the following, these substrates are called "neutron mirrors".

\subsection{State preparation mechanism}

All quantum mechanical experiments rely on a sufficient preparation of the incoming wave packet in a well-defined superposition of states. In our case, one has to find a mechanism to (1) filter all neutrons in undesired states or (2) transform neutrons in undesired states to the desired state. The practical realization of method (1) by a thin, rough polyethylene foil fixed to a flat glass surface is described in [14]. So far, the $q$ BOUNCE experiments have been using a variant of this proposal: we introduce a spatial region at the entrance, where we place a second, rough neutron mirror on top of the ordinary mirror. Neutrons in higher states are more likely to interact with the rough surface, and are scattered off the system. For the $q$ BOUNCE experiments, the choice of rough BK7 glass containing Boron as neutron mirrors is convenient. Adjusting the slit between the lower and upper mirror, the length of this system, and the roughness parameters of the upper mirror,

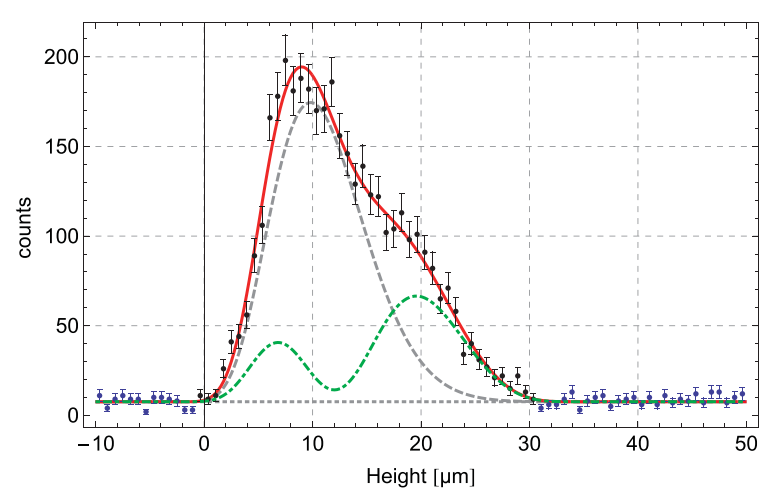

Figure 3. State preparation process [data set 3-14-283 from 2010]. With the help of a rough upper neutron mirror at a slit height of $\approx(29.3 \pm 0.9) \mu \mathrm{m}$, a preparation with a relative contribution of $(70 \pm 2) \%$ state $|1\rangle,(30 \pm 2) \%$ state $|2\rangle$ and no higher states occurring was achieved.

we can prepare a wavepacket, where only the first and second states are present. The plot on the right side of Fig. 1 illustrates the mechanism. This method does not allow for a preparation of a single ground state nor a single higher state. The state-preparation can be checked by measuring the probability density behind the state selector using a track detector with micron resolution (see Sect. 2.7). Such a measurement allows us to determine the relative contributions of all involved states. Figure 3 shows the results from the experimental campaign in 2010 $[15,16]$. A fit of the incoherent sum of states convoluted with a Gaussian filter to account for the spatial resolution of the detector results in a relative state contribution of $(70 \pm 2) \%$ in state $|1\rangle$ and $(30 \pm 2) \%$ in state $|2\rangle$. There is no evidence for higher states.

\subsection{Preparation of the velocity spectrum}

The incoming spectrum of the horizontal velocity component is rather broad, see Fig. 2. In order to allow for Rabi-type resonance spectroscopy, this spread has to be narrowed. To do so, we install two blades made of boronated Aluminium at a distance $\Delta x$ in front of the state selector. The blades are mounted at a distance $\Delta z_{A}$ and $\Delta z_{B}$ lower with respect to the slit of the state selector. As $\Delta z_{A, B}$ is much larger than the slit width $l$, and neutrons impinging on the rough upper mirror of the state selector are effectively scattered off, this system allows to constrain the horizontal velocity component $v_{x}$ of the neutrons:

$$
\frac{x}{\sqrt{\frac{2 \Delta z_{A}}{g}}} \leq v_{x} \leq \frac{x}{\sqrt{\frac{2 \Delta z_{B}}{g}}}
$$

Moreover, the spectrum can be determined in two ways: as an integral method, the variation of the height of one collimating blade and measuring the neutron flux behind the state selector allows to determine the cumulative velocity spectrum. The measurements from the campaign in 2010 are shown in Fig. 4. Secondly, since 2016, the $q$ BOUNCE collaboration decided to vary both collimating blades to desired cut-off velocities to determine the differential velocity spectrum directly [17]. 


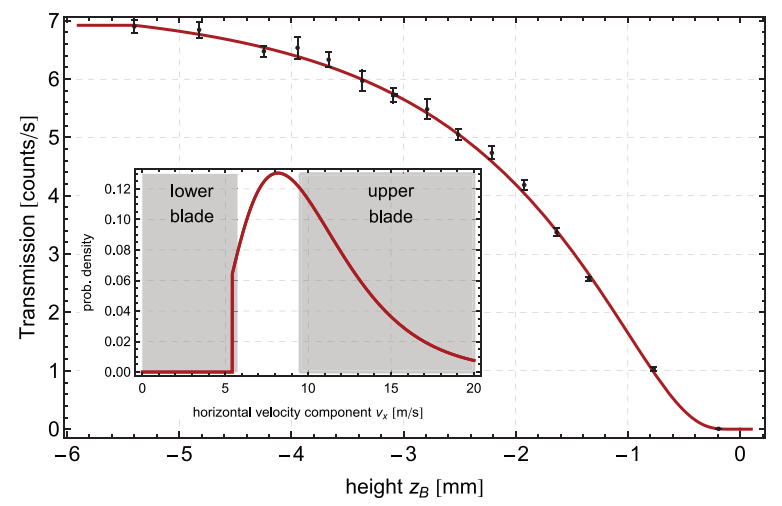

Figure 4. Preparation of the incoming velocity spectrum of UCNs [data set 3-14-283]. The data points show the transmission of neutrons while varying the lower collimating blade. The distance between collimating blades and the neutron mirrors was $180 \mathrm{~mm}$ long. The red theory curve shows a fit to this dataset. The inserts depicts the corresponding velocity spectrum. The shaded areas give the final adjustment of the upper and lower collimating blade.

\subsection{State transitions}

Resonance spectroscopy measurements rely on a technology to induce transitions between different states in a controlled and reproducible way. For the case of gravitational levels, the possibility of driving transitions was discussed for atoms [18] and neutrons [19,20]. From a practical point of view, only two different mechanisms seem feasible: First, the boundary condition might be oscillated sinusoidally with a well-defined frequency and amplitude. Second, one might make use of the magnetic moment of the free neutron, and induce vertical magnetic gradient fields.

While the GRANIT collaboration is about to implement the second possibility [21], the $q$ BOUNCE collaboration chose the first: transitions are induced by the mechanical oscillation of the neutron mirror with adjustable frequency and strength. For a practical realization, the neutron mirrors are mounted on piezodriven nanopositioning tables. These tables can be fed with an external oscillation signal, which modulates the piezo actuator length and hence leads to oscillating neutron mirror surfaces. Such controlled vibrations in a precision experiment may read scary to the reader. This is also true for the authors. Therefore, the oscillations are well-monitored using laser spectroscopy: A measurement device based on a commercial laser interferometer ${ }^{1}$ is able to scan the surface of the neutron mirrors in-situ and monitors continuously the oscillations relative to a reference point. A typical Fourier spectrum of an oscillating neutron mirror as well as parts of the raw spectrum are depicted in Fig. 5.

\subsection{Mind the step!}

The conceptually simplest resonance experiment is realized in three phases: First, the particle is prepared in a state $|p\rangle$. Then, transitions to state $|q\rangle$ are induced. Finally, the resulting wave function is analysed with respect to

\footnotetext{
${ }^{1}$ SP-2000-TR and SP-15000, produced by SIOS Meßtechnik $\mathrm{GmbH}$, Germany.
}

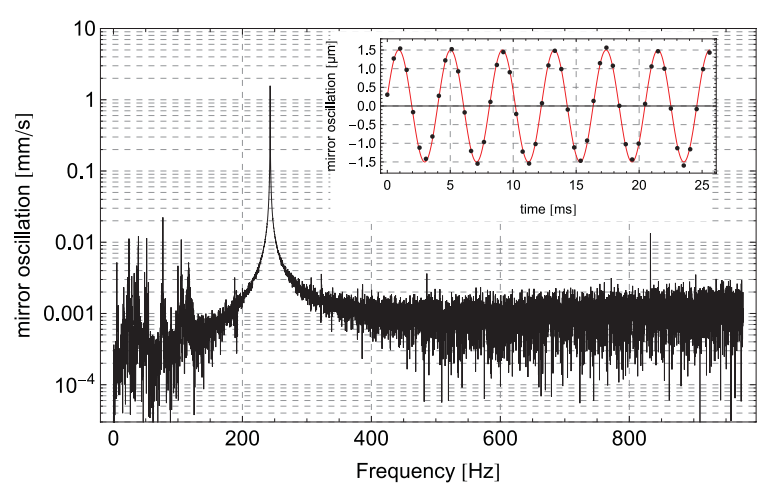

Figure 5. Oscillating mirror surface [data: exp. DIR94 from 2011]. The mirror is excited at a frequency of $243.5 \mathrm{~Hz}$ with a rather high oscillation amplitude of $\approx 3 \mu \mathrm{m}$ ). The resonance at $833 \mathrm{~Hz}$ is due to the rotation of the turbo pump.

state $|p\rangle$. For an in-flight UCN experiment, this requires the combination of at least three different setup parts, and the size of the wave function requires an absence of steps between the corresponding neutron mirrors to a level of a few $100 \mathrm{~nm}$ (depending on the precision). The $q$ BOUNCE collaboration solves this challenge by the help of capacitive sensors that scan the neutron mirror surface, and a feedback loop to the nanopositioning tables.

\subsection{UCN detection}

Finally, the UCNs have to be detected. As the available UCN flux is rather low, we designed a dedicated UCN counter based on a 10-Boron converter with an efficiency of $77 \%$ and a background rate of $(0.65 \pm 0.02) \cdot 10^{-3} 1 / \mathrm{s}$. In order to characterize the state preparation, we use CR39-based track detectors with a 10-Boron converter. Here, the spatial resolution is approx. $1.8 \mu \mathrm{m}$. A detailed description of the detector developments of the $q$ BOUNCE collaboration can be found in [22].

\section{Gravity Resonance Spectroscopy with qBOUNCE}

In the last decade, $q$ BOUNCE performed several experimental campaigns at the ultracold neutron installation PF2 of the ILL. In these campaigns, the collaboration implemented three generations of GRS setups.

\subsection{First generation: Rabi spectroscopy with damping}

The first campaign started in 2009 and includes the experiments 3-14-253 (5 days), TEST-1692 (15 days), 3-14-283 (50 days), and DIR94 (12 days) at the PF2/UCN beam position. The aim of these experiments was the first-time realization of GRS. For this purpose, the experimenters chose the technically simplest setup they could think of: instead of a three-phase experiment, the phases of state preparation, state transition and state analysis were implemented in a single neutron mirror system, consisting of a flat mirror on bottom and a rough mirror on top with a slit width of roughly $30 \mu \mathrm{m}$. The total setup (including both neutron mirrors) was oscillated with tunable frequency and amplitude. The basic idea was that the rough mirror on top reduces the transmission 


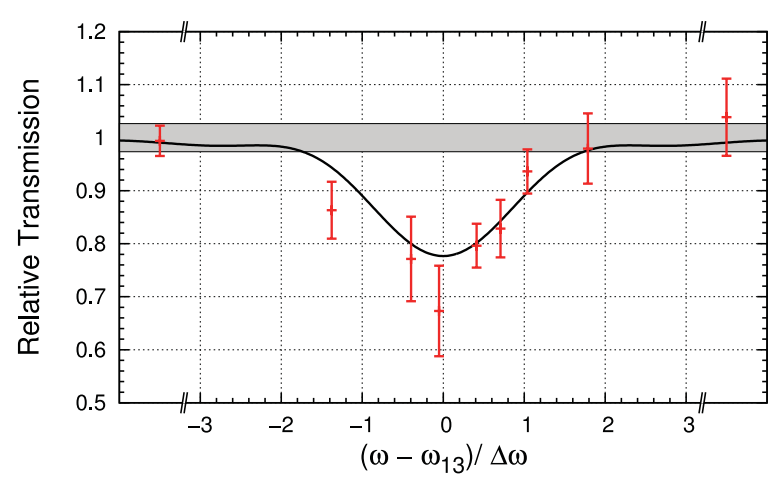

Figure 6. First realization of GRS in 2009 (source: [24], Fig 3b).

of higher states, and therefore introduces an asymmetry when exciting (for example) the $|1\rangle \rightarrow|3\rangle$ transition: In a naive picture, neutrons entering the system in state $|1\rangle$ could be excited to state $|3\rangle$ and scattered off the system. This effect would lead to a drop in countrate, compared to the measurement without oscillations. On the opposite, neutrons entering in state $|3\rangle$ undergo a state transition to state $|1\rangle$. This excess in rate is less than the drop of the opposite case, and hence leads to a measurable net effect. This system is also called "Rabi flopping with damping". From a technological point of view, the system consists only of two neutron mirrors, which minimizes the number of adjustable parameters, and avoids any steps. The additional absorber leads to a second boundary condition and hence introduces a shift of the transition frequencies, which potentially produces new sources of uncertainties. Therefore, the early experiments were meant to give an existence proof of Rabi spectroscopy. Nevertheless, searches for new physics in terms of zero-experiments can be very convenient with this setup. The theoretical description of the experiment is more complicated, but still has analytical solutions $[15,16]$. These solutions are approximate, because they simplify the multilevel-system to the sum of 2-level-systems and a 3-level-Cascadesystem. This simplification might result in small frequency shifts [23]. At the current level of precision, a systematic correction for this effect does not need to be taken into account. At the time of the first realization in 2009, the exact solution was not known to the experimentators, but the expected drop in count rate was observed with a statistical significance of $2.7 \sigma$. An invitation for an additional TEST-beamtime lead to the discovery of Gravity Resonance Spectroscopy with the observation of the $|1\rangle \rightarrow$ $|3\rangle$ transition with $5 \sigma$ significance [24], see Fig. 6 . This experiment was followed by a precision experiment in 2010, which led to the measurement of the transitions $|1\rangle \leftrightarrow|2\rangle \leftrightarrow|3\rangle,|1\rangle \rightarrow|3\rangle$ and $|1\rangle \rightarrow|4\rangle$ with a precision of roughly $1 \%$, see Fig. 7 .

In an extension of the beamtime, the $|1\rangle \rightarrow|3\rangle$ transition was measured with a magnetic guide field oriented parallel and anti-parallel to gravity, and a full spin-analysis of the detected neutrons. The experimental data was used to set limits on hypothetical chameleon fields, introduced by [25]. These scalar fields evolve cosmologically and couple to matter. Effectively, they emulate a cosmological constant and thus drive the observed accelerated expansion of the Universe. The basic concept is that chameleon fields acquire an effective mass that depends on the local matter density. In
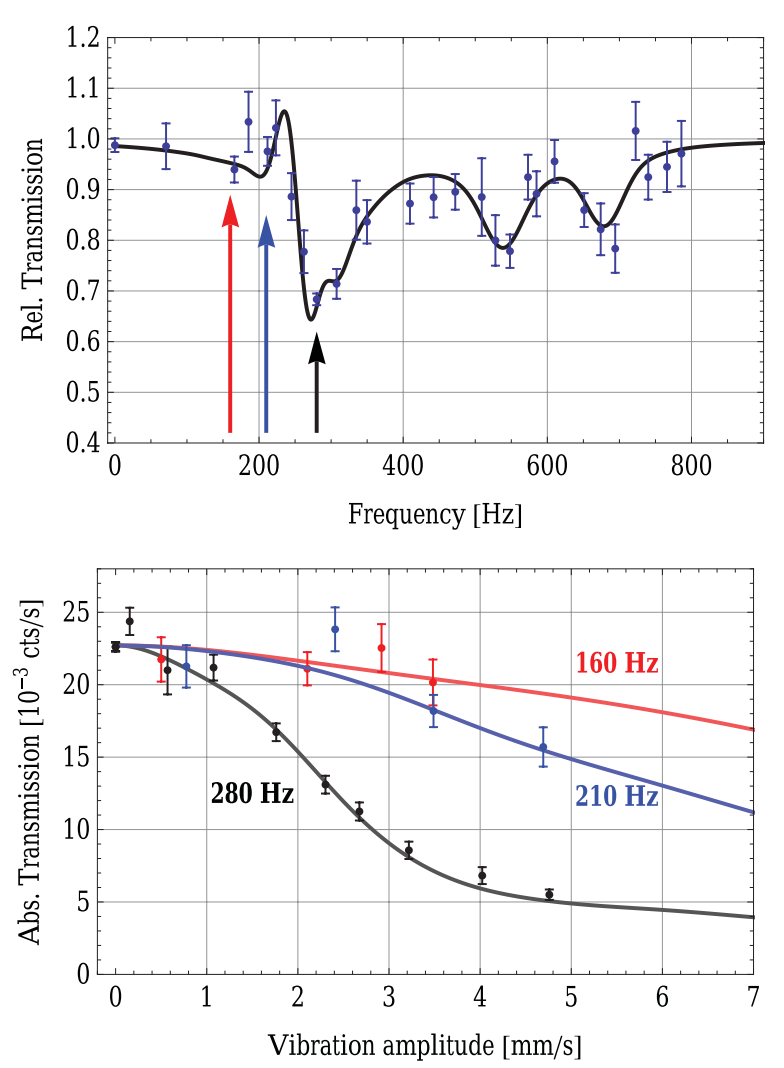

Figure 7. The experimental campaign in 2010 led to the first precision dataset (source: [16], Fig 2). The arrows in the upper frequency plot correspond to the frequencies, for which the transmission was scanned with respect of the oscillation strength, see lower plot. Due to the simplified setup and the resulting upper rough neutron mirror, these Rabi oscillations are damped and the transmission curve does not reach its inital value for oscillation strengths that correspond to $2 \pi$-flips.

high-density regions (like on Earth), this mass is large and the range of the force mediated by the particle is tiny the equivalence principle-violating force is therefore exponentially suppressed. Consequently, chameleon fields seem to be untestable using macroscopic bodies. On cosmological scales, however, the ambient mass density is very low, and the effective mass of the field is comparable to the present Hubble parameter. This results in an interaction range of the mediated force of up to several thousands of parsecs. While the Universe expands, its mass density decreases, leading to amplification of the field. This is how the chameleon field drives the observed accelerated expansion of the Universe. In 2011, Brax and Pignol discovered that chameleon theories could nevertheless be tested using table-top experiments [26]. They suggested using quantum states of ultracold neutrons (UCNs) in the Earth's gravitational field. The field equations and transition frequency shifts for the $q$ BOUNCE experiments are calculated in [27]. The experimental limits from the first generation experiment on the existence of chameleon fields as well as axion-like particles, a promising Dark Matter candidate, can be found in [16].

\subsection{Second generation: Full Rabi spectroscopy}

In the meantime, the $q$ BOUNCE collaboration addressed the still unsolved problem regarding steps between 

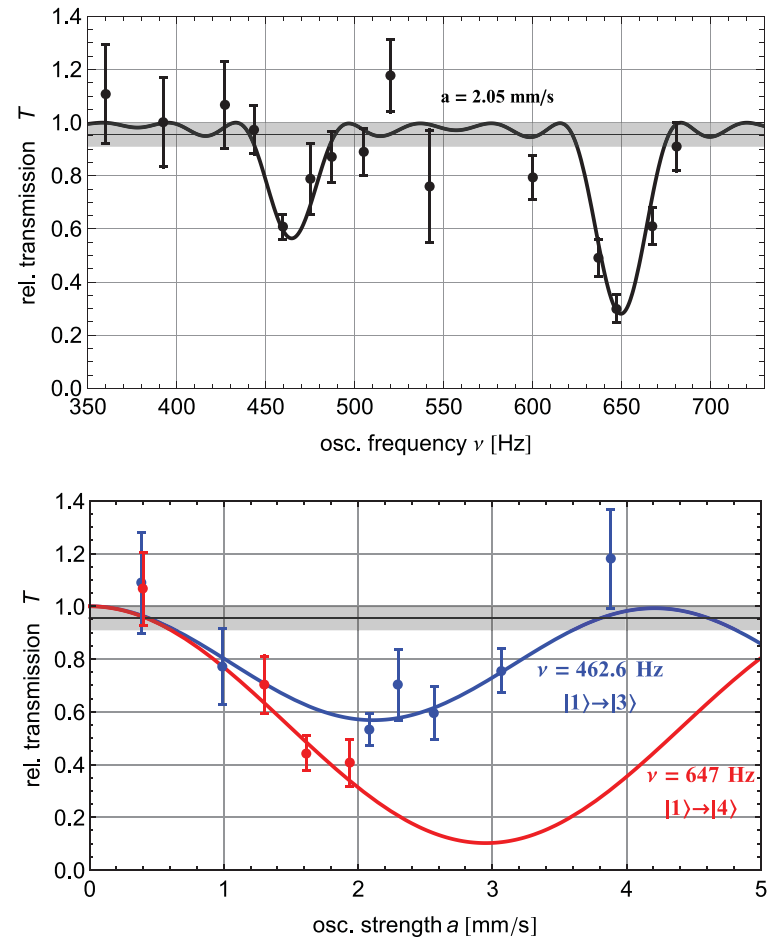

Figure 8. In 2012, the realization of Full Raboi spectroscopy succeeded (source: [28], Fig 2). The two dips correspond to the $|1\rangle \rightarrow|3\rangle$ and $|1\rangle \rightarrow|4\rangle$ transition. In the Rabi oscillation plot, the transmission reaches its initial value when inducing a $2 \pi$-flip.

different regions in our setup. Its solution paved the way for the implementation of a second generation of $q$ BOUNCE experiments at PF2: In 2012, during the experiments 3-14-305 (50 days) and 3-14-314 (25 days) at the PF2/UCN beam position, the collaboration succeeded in the realization of a "full" Rabi-type setup. Here, the state preparation, state transition and state analysis are performed separately in three regions, and steps between them are adjusted and held constant to a level of better than $0.5 \mu \mathrm{m}$. The major advantage of this system is that it makes the upper boundary condition in the region of state transition obsolete. Hence, the wave functions truly correspond to the case on the left side in Fig. 1 and the eigenenergies are only dependent on the reduced Planck constant $\hbar$, the neutron mass $m$, the zeros of Airy's function, and the local acceleration of the Earth $g$.

Major improvements in micropositioning and stabilization of the setup lead to a successful measurement of the $|1\rangle \rightarrow|3\rangle$ and $|1\rangle \rightarrow|4\rangle$ transition, see Fig. 8. The authors want to highlight the realization of a $2 \pi$-flip for the $|1\rangle \rightarrow|3\rangle$ transition, see the plot transmission vs. oscillation strength in Fig. 8. Here, an oscillation strength of roughly $4 \mathrm{~mm} / \mathrm{s}$ (which corresponds to a $2 \pi$-flip for UCNs with a velocity of $6.8 \mathrm{~m} / \mathrm{s}$ ) leads to a full revival of the transmission.

The relative error of the measurements is given by $\left(3 \cdot 10^{-3}\right)_{\text {stat }}$ and $\left(2 \cdot 10^{-3}\right)_{\text {sys }}$. Here, the main statistical error bar comes from the fact that the rate in our system was decreasing with time. A posteriori, we identified a broken oil-free pump and a hidden oil reservoir of an oilfree linear stage motor to be the direct cause. The decrease of the countrate was taken into account by two additional fit parameters and the hypothesis that the rate was decreasing exponentially. The uncertainty due to this procedure was the limiting factor for systematic effects [29]. The total error of $0.4 \%$ corresponds to an energy sensitivity of $2 \cdot 10^{-15} \mathrm{eV}$. Hypothetical Non-Newtonian gravity forces can be excluded to this level of sensitivity. Recently, the results were used to test the existence of Symmetron fields, which is another prominent Dark Energy scenario $[28,30]$.

\subsection{Third generation: Ramsey spectroscopy}

The most powerful resonance method known is Ramsey spectroscopy. This method is advantageous, because it is easily scalable, and furthermore works for a broad range of UCN velocities. The concept for Ramsey spectroscopy within $q$ BOUNCE is outlined in [31]. Its implementation also allows for new kinds of experiments, for example the search for a tiny charge of the free neutron [32]. In order to implement this method, the $q$ BOUNCE collaboration redesigned its spectrometer in 2015. This includes the enlargement of the vacuum chamber and the pumping system, the introduction of a double-layer $\mu$-metal-shielding against the Earth's magnetic field, and the redesign of the neutron guide system to gain neutron flux [12]. The spectrometer was installed and commissioned in 2016 and data-taking is on-going since 2017. The $q$ BOUNCE collaboration devotes a specific article within this volume to the developments of Ramsey spectroscopy of gravitationally bound quantum states of ultracold neutrons [17].

\section{Conclusion}

As discussed in the first part of the article, our picture of the Universe seems incomplete, and still contains various mysteries. Here, (also) lab experiments can contribute to address these questions. In particular, gravitationally bound quantum states of ultracold neutrons are very sensitive and efficient probes, and offer the possibility to perform precise resonance spectroscopy measurements to draw conclusions about gravity at short distances. The $q$ BOUNCE collaboration developed and realized such a resonance technique, and named it Gravity Resonance Spectroscopy. Results of the first two generations of experiments were used to test the existence of hypothetical chameleon and symmetron Dark Energy, and to search for Dark Matter realized as axion-like particles. Currently, the third generation experiment focusses on Ramseyspectroscopy. Data-taking is on-going at PF2, the ultracold neutron installation at the Institut Laue-Langevin.

The authors would like to thank M. Faber (TU Wien), G. Pignol (LPSC, Grenoble), D. Dubbers (University of Heidelberg), U. Schmidt (University of Heidelberg), T. Lauer (University of Mainz, FRM II, now at Movatec), H. Lemmel (TU Wien, ILL) for useful discussions. We also would like to thank all undergraduate students for their valuable input, which contributed to the success of our experiments. We thank D. Seiler (TU München) for preparing spatial resolution detectors for several years, R. Stadler (University of Heidelberg) for preparing various rough mirror surfaces. We thank T. Brenner (ILL) and R. Ziegler (University of Heidelberg) for their outstanding technical support.

The $q$ BOUNCE experiment would not have been possible without appropriate funding. We gratefully acknowledge support from the Austrian Science Fund (FWF) under Contract No. I529N20, No. I531-N20, No. I862-N20, No. P31702-N27, W1252N27 (DK-PI) and the German Research Foundation (DFG) 
as part of the Priority Programme (SPP) 1491 "Precision experiments in particle and astrophysics with cold and ultracold neutrons", the DFG Excellence Initiative "Origin of the Universe", and DFG support under Contract No. Ab128/2-1. We also gratefully acknowledge support from the French Agence Nationale de la Recherche (ANR) under Contract No. ANR-2011-ISO4-007-02, Programme Blanc International'sIMI4-Physique.

\section{References}

[1] ATLAS-collaboration, Phys. Lett. B 716, 1 (2012)

[2] CMS-collaboration, Phys. Lett. B 716, 30 (2012)

[3] N. Arkani-Hamed, S. Dimopoulos, G. Dvali, Phys. Rev. D 59, 086004 (1999)

[4] G. Breit, Phys. Rev. 32, 273 (1928)

[5] P.W. Langhoff, Am. J. Phys. 39, 954 (1971)

[6] R.L. Gibbs, Am. J. Phys. 43, 25 (1975)

[7] A. Steyerl, Phys. Lett. B 29, 33 (1969)

[8] V.I. Lushchikov, Y.N. Pokotilivski, A.V. Strelkov, F.L. Shapiro, ZhETF Pis. Red. 9, 40 (1969)

[9] V.I. Lushchikov, A. Frank, JETP Lett. 28, 559 (1978)

[10] V.V. Nesvizhevsky, H.G. Börner, A.K. Petukhov, H. Abele, S. Baeßler, F.J. Ruess, T. Stöferle, A. Westphal, A.M. Gagarski, G.A. Petrov et al., Nature 415, 297 (2002)

[11] J. Gea-Banacloche, Am. J. Phys. 67, 776 (1999)

[12] T. Rechberger, Ph.D. thesis, Technische Universität Wien (2018), https://repositum.tuwien.ac. at/urn:nbn:at:at-ubtuw:1-119385 TR-Diss

[13] S. Döge, Ph.D. thesis, Technische Universität München (2019)

[14] V. Nesvizhevsky, H. Börner, A. Gagarski, G. Petrov, A. Petukhov, H. Abele, S. Bäßler, T. Stöferle, S. Soloviev, Nucl. Instr. Meth. A 440, 754 (2000)

[15] T. Jenke, Ph.D. thesis, Technische Universität Wien (2011), http://www.ub.tuwien.ac.at/ diss/AC07811416.pdf

[16] T. Jenke, G. Cronenberg, J. Burgdörfer, L.A. Chizhova, P. Geltenbort, A.N. Ivanov, T. Lauer, T. Lins, S. Rotter, H. Saul et al., Phys. Rev. Lett. 112, 151105 (2014)
[17] R. Sedmik et al., EPJ Web of Conf. 219, 05004 (2019)

[18] H. Wallis, J. Dalibard, C. Cohen-Tannoudji, Appl. Phys. B 54, 407 (1992)

[19] V. Nesvizhevsky, K. Protasov, Quantum States of Neutrons in the Earth's Gravitational Field: State of the Art, Applications, Perspectives (Nova Science Publishers, Inc., New York, 2006), ISBN 1-59454670-3

[20] A. Landry, M.B. Paranjape, Phys. Rev. D 93, 122006 (2016)

[21] G. Pignol, S. Baeßler, V. Nesvizhevsky, K. Protasov, D. Rebreyend, A. Voronin, Adv. High Energy Phys. 2014, 628125 (2014)

[22] T. Jenke, G. Cronenberg, H. Filter, P. Geltenbort, M. Klein, T. Lauer, K. Mitsch, H. Saul, D. Seiler, D. Stadler et al., Nucl. Instr. Meth. A 1 (2013)

[23] S. Baeßler, V.V. Nesvizhevsky, G. Pignol, K.V. Protasov, D. Rebreyend, E.A. Kupriyanova, A.Y. Voronin, Phys. Rev. D 91, 042006 (2015)

[24] T. Jenke, P. Geltenbort, H. Lemmel, H. Abele, Nat. Phys. 7, 468 (2011)

[25] J. Khoury, A. Weltman, Phys. Rev. Lett. 93, 171104 (2004)

[26] P. Brax, G. Pignol, Phys. Rev. Lett. 107, 111301 (2011)

[27] A.N. Ivanov, R. Höllwieser, T. Jenke, M. Wellenzohn, H. Abele, Phys. Rev. D 87, 105013 (2013), 1207.0419

[28] G. Cronenberg, P. Brax, H. Filter, P. Geltenbort, T. Jenke, G. Pignol, M. Pitschmann, M. Thalhammer, H. Abele, Nat. Phys. 14, 1022 (2018)

[29] G. Cronenberg, Ph.D. thesis, Technische Universität Wien (2015), http://repositum.tuwien.ac. at/obvutwhs/download/pdf/1643386?

[30] P. Brax, M. Pitschmann, Phys. Rev. D 97, 064015 (2018)

[31] H. Abele, T. Jenke, H. Leeb, J. Schmiedmayer, Phys. Rev. D 81, 065019 (2010)

[32] K. Durstberger-Rennhofer, T. Jenke, H. Abele, Phys. Rev. D 84, 036004 (2011) 